\title{
Automated load balancing in the ATLAS high-performance storage software
}

\author{
Fabrice Le Goff and Wainer Vandelli \\ On behalf of the ATLAS Collaboration \\ CERN
}

\begin{abstract}
ATLAS [1] is one of the general purpose detectors observing proton-proton collisions provided by the LHC [2] at CERN. The ATLAS Trigger and Data Acquisition (TDAQ) system [3] is responsible for conveying the event data from the detector up to a permanent mass-storage system provided by CERN. This work focuses on the Data Logger system which lies at the end of the data flow path in the TDAQ system. The Data Logger is a transient storage system recording the selected event data on hard drives before transferring them to permanent storage where they are available for offline analysis.
\end{abstract}

\section{Data Logger and Workload Distribution}

The purpose of the Data Logger is to decouple the online from the offline operations. It enables ATLAS to cope with disruptions of the permanent storage service. Its tasks are to write selected event data to non-volatile storage and to transfer them to permanent storage outside of the ATLAS facility.

In terms of hardware the Data Logger is a scale-out system currently consisting of four local-attached high-performance storage solutions sporting two head servers each. It can easily be upgraded to provide more storage space or more bandwidth. The system comprises almost five hundred drives with a total usable space of $430 \mathrm{~TB}$. It is able to provide a total of $8 \mathrm{~GB} / \mathrm{s}$ of concurrent read and write operations.

These servers execute a distributed multi-threaded in-house application that receives selected event data over two $10 \mathrm{GbE}$ network links and writes them to disks in an organized file scheme. It also computes a file-by-file Adler32 [4] checksum. The application is completely data-driven, therefore its workload is entirely determined by the data composition and indirectly by the trigger configuration.

The trigger system classifies the events in classes called streams. Each event can be assigned to one or several streams. Each event is also associated with a luminosity block (lumiblock), a time interval for which the detector's operation conditions are considered constant. The typical duration of a lumiblock is $60 \mathrm{~s}$. The application writes all events of the same stream and lumiblock to a dedicated file. Figure 1 shows a typical stream throughput distribution. As shown, the application workload is very unbalanced.

Figure 2 shows relevant parts of the application threading model. Each instance of the application spawns a configurable set of input threads to handle the network communications and receive the event data. Another number of output 


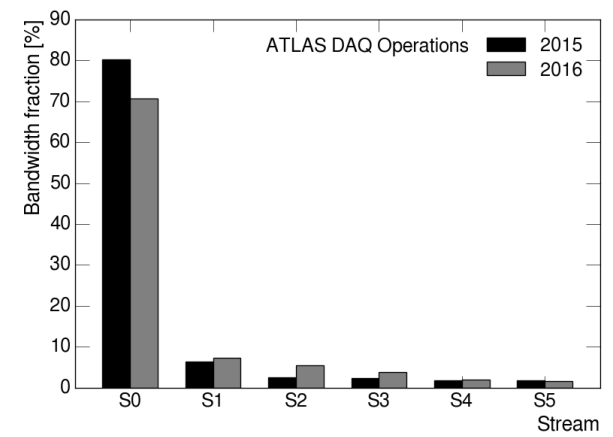

Figure 1: Comparison of 2015 and 2016 stream throughput distribution for typical operations. Only the six major streams out of $25+$ are shown. It shows the intrinsically non-uniform nature of the application workload and its evolution between 2015 and 2016.

threads compute the checksum and write the data to file. An external library provides both the file format and the checksum computation. The file format requires the application to write files sequentially. Between these two sets of threads several components dispatch the incoming events among the available output threads. In order to enforce sequential writing, these components assign each file to one thread upon file creation. This association cannot be changed and every event intended for this file must be processed by this thread. Therefore the streams cannot be divided into smaller chunks processed in parallel to distribute the stream processing.

The current file-to-thread assignment policy is based on a trivial round-robin scheduling: each new file is assigned to the next thread in a circular fashion. This policy is robust and has very little overhead. Since at any particular moment in time there are more active streams than available output threads, one thread handles several streams. Moreover the events reach the application with no specific order. As a consequence different files are assigned to the same thread randomly.

As the application is processing real-time data, one single overloaded thread will limit the whole application performance. Specifically the assignment of major streams together to the same thread could overload this thread and then degrade the whole application throughput. As a consequence with the round-robin assignment policy the instantaneous application performance is not predictable.

\section{New Assignment Policy}

Between 2015 and 2016 the peak system writing throughput more than doubled going from $\approx 1.4 \mathrm{~GB} / \mathrm{s}$ to $\approx 3.2 \mathrm{~GB} / \mathrm{s}$. The application was, therefore, running closer to its saturation point. Figure 1 and shows the evolution of the stream throughput distribution for typical ATLAS operations between 2015 and 2016. As one can see the relative difference between the major streams is less in 2016. 


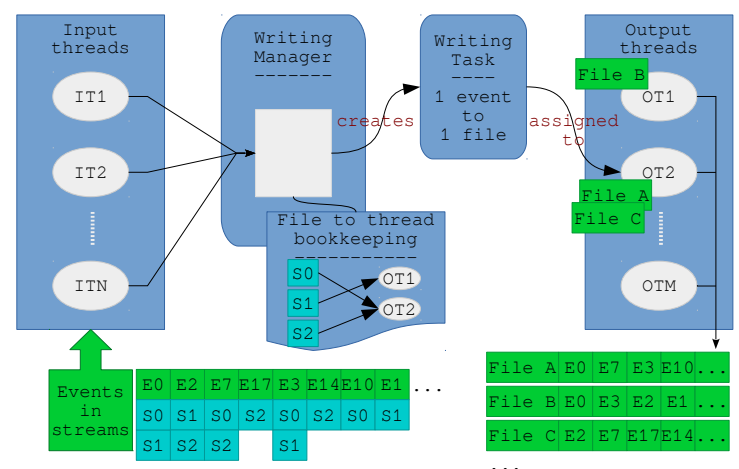

Figure 2: Overview of the threading model of the Data Logger application. Input threads handle network communications. The middle components dispatch the events among the output threads. Output threads compute the data checksum and write data to disks.

For these two reasons the random assignment of major streams together to a thread would actually degrade the application performance. Synthetic tests confirmed the performance degradation upon occurrence of these conjoint assignments: $-8 \%$ for the two major streams together, and $-12 \%$ for the three major streams together. In order to re-establish the application performance, a smarter load-balancing algorithm, sensitive to the application conditions, was needed.

A weighted policy was designed to take assignment decisions based on a load for threads: a new file is assigned to the thread with the lowest load. The thread load shall represent its current activity. This will allow optimally distributing the application workload among the threads. The thread load is computed from the amount of data processed during a configurable time window. The determination of the operational value for this period will be a trade-off between the desired sensitivity to condition changes and the accuracy of the decisions. In the same manner a load is computed for each stream. Upon assignment the load of the stream is added to the load of the thread. Therefore the thread load reflects immediately the assignment without waiting for real-time data to accumulate. This will ensure that streams with significant throughput will not be handled by the same thread.

Figure 3 shows the algorithm behavior by plotting the thread loads computed for a 5-second period and showing an example of assignments of the three major streams to different threads. The thread loads evolve according to real-time data processing. Upon assignment the thread load receives a boost corresponding to the assigned stream load. The probability of the chosen thread to be selected again for the next new stream is then inversely proportional to the load of the assigned stream, no matter how close the next decision is taken.

The solution was first tested in a controlled environment using an emulated data flow. Emulation parameters were extracted from actual 2016 monitoring data. No wrong assignment decisions were taken during operating periods of more than 40 hours. It was checked that the overhead added by the new al- 


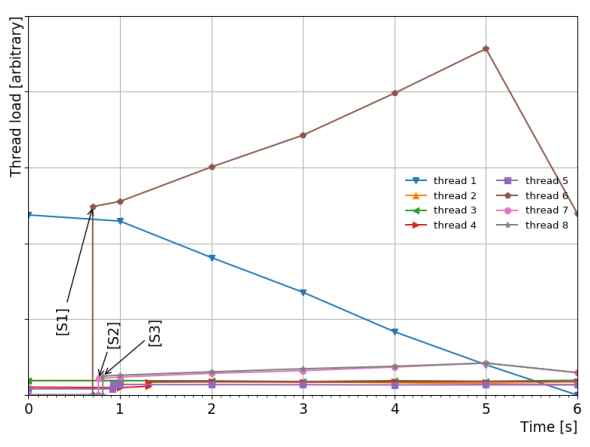

(a)

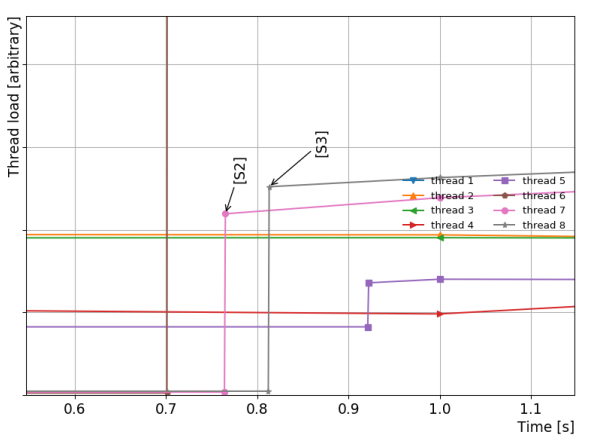

(b)

Figure 3: Thread workloads as a function of time (left) and zoom around the assignment decisions (right). Each line represents the load for a different thread computed for a 5second period. Annotations mark the assignment of the three major streams to threads.

gorithm does not impair the performance of the application. Results showed a slight improvement of $2 \%$ even excluding wrong assignments. The application was then tested on the production infrastructure and has since been included in all ATLAS commissioning periods.

\section{Conclusion}

The Data Logger system of the ATLAS TDAQ system is a key component enabling the decoupling of the online and offline operations. Its workload is essentially unbalanced and cannot be fairly distributed. In 2016 new operation conditions required a new workload distribution strategy. A weighted policy was designed to be sensitive and self-adaptive to evolving operational conditions. It has been validated on both test and production systems. It proved to restore the application performance predictability. This development is now part of the TDAQ system for the 2017 data-taking period.

\section{References}

1. ATLAS Collaboration. Performance of the ATLAS detector using first collision data. JHEP, 09:056, 2010.

2. Lyndon Evans and Philip Bryant. Lhc machine. Journal of Instrumentation, 3(08):S08001, 2008.

3. The ATLAS TDAQ Collaboration. The atlas data acquisition and high level trigger system. Journal of Instrumentation, 11(06):P06008, 2016.

4. P. Deutsch, Aladdin Enterprises, and J-L. Gailly. Zlib compressed data format specification version 3.3. 1996. 